\title{
Simulating alphabet recitation under thalamic lesions
}

\author{
Martin D. Pham ${ }^{1}$, Terrence C. Stewart ${ }^{2}$, Suzanne Tyas ${ }^{3}$, Randy A. Harris ${ }^{4}$ \\ ${ }^{1}$ The Centre for Computational Medicine, The Hospital for Sick Children, Canada \\ ${ }^{2}$ Applied Brain Research, Canada \\ ${ }^{3}$ The School of Public Health and Health Systems, University of Waterloo, Canada \\ ${ }^{4}$ Department of English Language and Literature, University of Waterloo, Canada \\ https://doi.org/10.36505/ExLing-2019/10/0039/000401
}

\begin{abstract}
We utilize the Semantic Pointer Architecture, a neurocognitive architecture in order to model language impairments. Constructed is a spiking neural network to investigate the effect of neural deficits in the basal ganglia and thalamus on the retrieval of an ordered sequence of unique symbols. The model includes four subnetworks: associative memory, working memory, basal ganglia and thalamus. A lesion is simulated by reducing the number of available neurons in the thalamus and attenuating its input from the basal ganglia. The model remains mostly successful in the ordered retrieval of the alphabet but 'stutters': working memory 'forgets' the current letter and 'steps back' several letters before continuing correctly.
\end{abstract}

Key words: spiking neural network, language, lesions

\section{Introduction}

The model makes use of the Neural Engineering Framework (NEF) (Eliasmith, 2004) methodology and Semantic Pointer Architecture (SPA) (Eliasmith, 2013) to construct a spiking neural network in the neural simulator Nengo (Stewart, 2009). NEF is an approach to neural simulation with an emphasis on three principles: neural representation, transformation and dynamics; each principle related to a mathematical formulation. SPA is an approach to cognitive modelling that represents higher-level cognitive concepts within the NEF as high-dimensional vectors.

\section{Neural Engineering Framework}

The NEF is a framework for understanding neurobiological systems within a rigorous mathematical formulation consisting of three principles relating the former to the latter:

\section{Representation}

Neuronal activations in a population of neurons encode a time-dependent vector signal nonlinearly as a spike train and decode as a weighted linear combination.

ExLing 2019: Proceedings of $10^{\text {th }}$ International Conference of Experimental Linguistics, 25-27 September 2019, Lisbon, Portugal 
158 M.D. Pham et al.

\section{Transformation}

Decoding of an input signal can estimate some nonlinear transformations.

\section{Dynamics}

Neural representations can be considered as control theoretic state variables. Neural dynamics can thus be described and analyzed mathematically using control theory.

The NEF may be used to design spiking neural networks to model perceptual, motor and cognitive systems. The software Nengo implements these principles to construct biologically plausible neural systems.

\section{Semantic pointer architecture}

The SPA is a framework that treats higher-level cognitive functions using neural representations carrying partial semantic content that may be composed into complex representational structures using basic mathematical operations. By representing concepts as high-dimensional vectors within the NEF, concepts may be 'bound' together and 'combined' within a scene using the mathematical operations of circular convolution $\left(^{*}\right)$ and vector addition $(+)$.

Consider an example making use of the subject-verb-object structure. Given a vocabulary of neurally representable concepts

\{CAT, DOG, RED, TALL, SMELL, SUBJECT, VERB, OBJECT\},

they may be used to describe the scene:

$$
\text { SMELL*VERB + TALL*CAT*SUBJECT + RED*DOG*OBJECT }
$$

representing a tall cat smelling a red dog. Note that the order of binding and combining does not matter due to the associative and commutative properties of convolution and addition. Querying for the object of the scene can be done by convolution with the inverse (i.e. 'unbinding') of OBJECT:

$$
\begin{aligned}
& \text { OBJECT }^{-1} * \text { (TALL*CAT*SUBJECT } \\
& \text { RED } \left.^{*} \text { DOG }^{*} \text { OBJECT }\right)
\end{aligned}
$$

and would return in the neural representation a vector close to RED*DOG.

\section{Model and discussion}

The model consists of four subnetworks implemented in the neural simulator Nengo: (1) associative memory, (2) working memory, (3) basal ganglia, (4) thalamus. The English alphabet is encoded into associative memory and only retrieved into working memory when given a cue to recite that is processed by 
the basal ganglia and routed through the thalamus. Figure 1 presents a diagram of the connections between different subnetworks.

The associative memory network encodes the sequential pattern of the alphabet. The SPA vocabulary is defined to be the 26 letters of the English alphabet and an additional concept for prompting recitation. Encoded in the heteroassociative memory is the relationship of the first 25 letters to their respective following letter, as well as the relationship of prompting recitation to the first letter $A$. The subnetwork is configured to be a winner-take-all network: given a semantic pointer, only one associated semantic pointer is recalled (i.e. $P R O M P T \rightarrow A, A \rightarrow B, B \rightarrow C, \ldots, \mathrm{Y} \rightarrow \mathrm{Z})$.

The working memory network is a simple neural population with a recurrent connection that acts as a feedback loop such that the desired target semantic pointer is retained. Mathematically, this is equivalent to the high-dimensional integration over time of a vector (representing a semantic pointer) with a control that gates input signals.

The basal ganglia network is responsible for winner-take-all action selection and consists of component subnetworks: striatum, subthalamic nucleous, globus pallidus internal, globus pallidus external, substantia nigra pars reticulata. The selected action is based on a context-dependent utility signal for each action. Two actions are defined for the model:

Recite If working memory is neurally representing any letter from the alphabet then working memory begins to recall from associative memory the next letter.

End If working memory is neurally representing the letter $Z$ then working memory begins to forget everything and resets.

The thalamus network routes the output of the basal ganglia to working memory. The firing within the thalamus represents the desired action selected and propagates the signal forward.

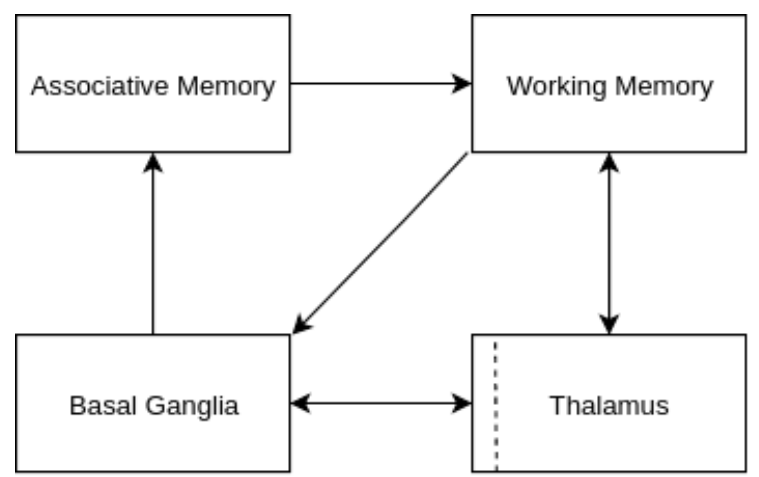

Figure 1. Model connectivity between subnetworks. Note that the signal towards the dashed line is attenuated under simulated lesions. 
Lesions are simulated by reducing the neurons within the thalamus from 3800 to 3010 . The number of neurons in both associative memory, working memory, and basal ganglia is fixed at 1300, 3200 and 4200, respectively. Figure 2 presents semantic pointer activation (representing letters) within working memory over simulation time for both unlesioned and lesioned recitations. The repetition of light blue ('F'), blue (' $G$ '), green (' $\left.\mathrm{H}^{\prime}\right)$ and orange (' $\mathrm{I}$ ') peeks seen in the right graph show that the model incorrectly recites the alphabet in a structured way: the order of letters in the alphabet are preserved before continuing correctly.

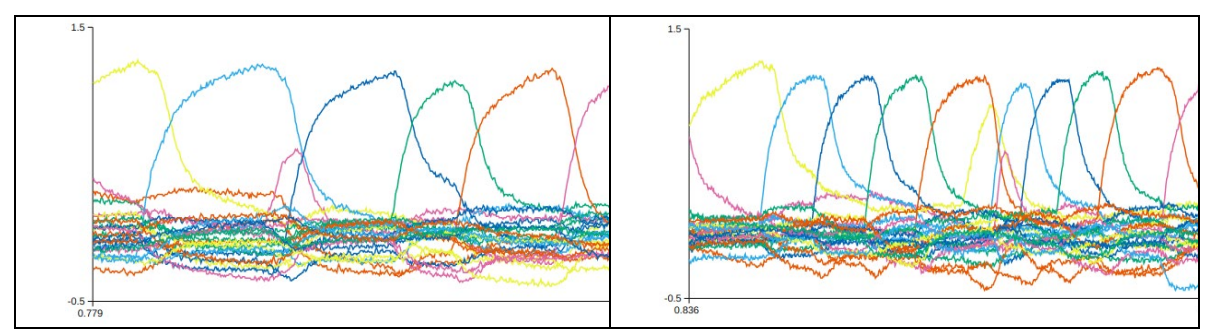

Figure 2. Recitation with (right) and without (left) simulated lesion. Without lesion: 'EFGHIJ'. With lesion: 'EFGHIFGHIJ'.

\section{Conclusion and future directions}

This exploratory experiment suggests that spiking neural networks may be useful in modelling neurodegeneration by reducing the size of neural populations within subnetworks to simulate lesions. Additionally, the use of semantic pointers to represent higher-order concepts within a rigorous mathematical framework allows for the modelling of cognitive tasks. Further directions include implementing subnetworks that better represent regions of the brain in order to produce more biologically plausible simulations and to model language within the Semantic Pointer Architecture.

\section{References}

Eliasmith, C., Anderson, C.H. 2004. Neural Engineering: Computation, Representation, and Dynamics in Neurobiological Systems. MIT Press.

Eliasmith, C. 2013. How to build a brain: A neural architecture for biological cognition. Oxford University Press.

Stewart, T.C., Tripp, B., Eliasmith, C. Python scripting in the Nengo simulator. Frontiers in neuroinformatics 3,7 . 